\title{
Theory for Articulated Double-Flap Wavemakers in Water of Constant Depth
}

\author{
by Jae Min Hyun*
}

\begin{abstract}
Extending the theoretical work of Biésel, a preliminary study is made of dynamic characteristics of an articulated double-flap wavemaker. The underlying motivation of utilizing a double-flap waveboard is based on its effectiveness in reducing the local disturbances in the immediate vicinity of the waveboard, which is demonstrated through decreasing inertia pressures. The additional degree of freedom in the double-flap waveboard movements is shown to be capable of encompassing a wider range of wavelength that can be satisfactorily generated in a given tank. The inertia pressure is reduced significantly by use of double-flap wavemakers, especially when short waves are generated. The usual wavemaker performance curves are computed for double-flap wavemakers. Comparison with the results of a single-flap wavemaker characteristics reveals that considerable improvement in performance curves is feasible by selecting a suitable combination of design parameters.
\end{abstract}

\section{Introduction}

The classical wavemaker theory of Havelock was further refined by Biésel ${ }^{1)}$, chiefly in reference to piston-type and flap-type wavemakers in laboratory use. The Biésel's analysis may be applied to a variety of wavemakers ${ }^{2)-5}$, with necessary modification in the contact surface boundary condition pertinent to the wavemaker in question. The Biésel's analysis is based on the first-order linearized hydrodynamic equations of two-dimensional irrotational motions in prismatic channels of rectangular cross section.

Partial experimental verifications of the first-order theory were reported by Ursell, Dean, and $\mathrm{Yu}^{6)}$ for the case of a piston-type wavemaker, and by Taniguchi and Shibata ${ }^{7)}$ for a flap-type wavemaker.

Aside from the problems involved in factual mechanical fabrication, the greater part of unfavorable features of a wavemaker performance, such as waveform distortions very near the waveboard and excessive hydrodynamic loads on the waveboard, may basically be attributed to the added mass effect of the waveboard motions ${ }^{2}$. The added mass effect may be explained in terms of the inertia pressure, $p_{i}$, which represents the local disturbances in the immediate vicinity of the waveboard. The local disturbances are created by, among other things, the difference between the waveboard

* Korea Research Institute of Ship and Ocean P.O. 131, Dong Dae Mun, Seoul, KOREA motions and the actual water particle movements as desired waves are generated.

Whereas the wave (or normal) pressure $p_{w}$, which is the hydrodynamic pressure far down the channel, is primarily a function of $\lambda / h$ (the ratio of wavelength $\lambda$ to the tank water depth $h$ ), the inertia pressure $p_{i}$ strongly depends on the waveboard particulars as well as on $\lambda / h$.

The common flap-type wavemaker ${ }^{233) 577)}$ is normally equipped with a single-flap rigid straight wabeboard with its lower end hinged somewhere in the middle of the wall. It was found ${ }^{2}$ that the flap size $f_{s} / h$ is a crucial design parameter in predicting a single-flap wavemaker performance. In general, if the flap size is moderate or large (say $f_{s} / h \geq 0.5$ ), the relative magnitude of $p_{i}$ is smaller than $p_{w}$ when long waves (say $\lambda / h \geq 2.0$ ) are generated, but in the case of short waves $p_{i}$ increases to the extent of almost overwhelming $p_{w}$ [Ref. (2)]. Consequently, if the main purpose of a single-flap wavemaker is to generate short waves (e.g., $\lambda / h \leqq$ 0.5 ), then the proper size of the flap should be quite small $\left(f_{s} / h \cong 0.25\right)$ with a view towards entailing much reduced inertia pressure. From the standpoint of minimizing the inertia pressure (or the local disturbances), it may be stated that small flaps are preferable when generating short waves, and that flaps of increased sizes should be chosen in order to generate moderate and long waves. In the extreme limit of very long waves $(\lambda / h \gg 1)$, the most appropriate wavemaker would be the one with a piston-type waveboard $\left(f_{s} / h=1.00\right)$ executing purely 
horizontal motions with uniform stroke ${ }^{1)}$.

In practice, therefore, when a single-flap wavemaker of fixed flap dimension is installed in a tank, there will be a wavelength zone in which the installed wavemaker is most effective. As a result, it is rather difficult to satisfactorily generate waves covering a wide range of wavelength by means of a single-flap wavemaker.

In an attempt to devise a flap-type wavemaker which can encompass a wider range of wavelength, we consider an extension of the Biésel's analysis to an articulated double-flap wavemaker, each sectional flap of which is controlled separately. In a hypothetical situation when the waveboard is partitioned into infinitely many separately-controlled sectional flaps, this could be envisioned as an ideal membranelike waveboard ${ }^{1)}$ whose motions may approximate the actual water particle movements with higher precision.

As a sequel to the previous analysis ${ }^{2)}$ of a singleflap wavemaker performance, a study is made in this paper of dynamic characteristics of an articulated double-flap wavemaker in water of constant depth. An examination of a double-flap wavemaker can also serve as a first step toward further investigations into multi-flap wavemakers.

\section{General Formulation}

Let us consider two-dimensional irrotational motions of an inviscid incompressible fluid in a semi-infinite channel $(0 \leq x<\infty)$ with rectangular cross section of width $B$ and of constant water depth $h$. The coordinate system and the schematic diagram of an articulated double-flap wavemaker arrangement is depicted in Fig. 1.

The velocity potential $\phi(x, y, t)$ for small-amplitude waves generated by a simple-harmonic waveboard motion about the mean position $x=0$ is governed by the Laplace equation

$$
\frac{\partial^{2} \phi}{\partial x^{2}}+\frac{\partial^{2} \phi}{\partial y^{2}}=0 \quad \text { in } \quad 0 \leq x<\infty, \quad 0 \leq y \leq h,
$$

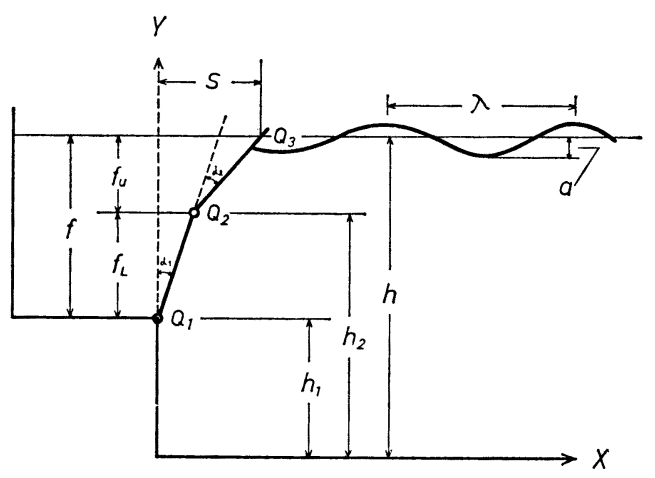

Fig. 1 Coordinate system and schematic diagram of an articulated double-flap wavemaker. Figure is exaggerated for distinctness. with the associated boundary conditions ${ }^{1)}, 6$ )

$$
\begin{array}{ll}
\frac{\partial \phi}{\partial y}=0 & \text { on } \quad y=0 \text { (tank bottom), } \\
\omega^{2} \phi-g \frac{\partial \phi}{\partial y}=0 & \text { on } \quad y=h \text { (free surface), } \\
\frac{\partial \phi}{\partial x} \cong \omega \xi(y) \cos \omega t & \text { on } \quad x=0 \text { (waveboard), }
\end{array}
$$

and, at large values of $x$, the solution is chosen to represent waves propagating towards $x=\infty$, thus the radiation condition at $x=\infty$ is satisfied. The usual waveboard contact surface boundary condition, Eq. (4), may be invoked ${ }^{1)}$ here, assuming that the maximun flap angles $\alpha_{1}$ and $\alpha_{2}$ are small, and, subsequently, the particle displacements from the mean waveboard position $x=0$ are also small. The upper flap $\overline{Q_{2} Q_{3}}$ is rotating about the end point $Q_{2}$ of the lower flap $\overline{Q_{1} Q_{2}}$, but it is presumed that both flaps are oscillating with the same frequency $\omega$. In addition, we treat in this paper the case when the two flaps are oscillating in phase. If there is a nonzero phase lag between the relative motions of the two flaps, a term associated with $\sin \omega t$ would be included in the right-hand side of Eq. (4).

The velocity potential $\phi(x, y, t)$ for this problem is $^{1), 6)}$

$$
\begin{aligned}
\phi= & -\frac{\omega}{k} A \cosh k y \sin (\omega t-k x) \\
& -\sum_{n=1}^{\infty} \frac{\omega}{k_{n}} A_{n} \cos k_{n} y \exp \left(-k_{n} x\right) \cos \omega t,
\end{aligned}
$$

in which $k$, the wavenumber of a generated wave of wavelength $\lambda(\equiv 2 \pi / k)$, is the positive real root of the dispersion relation $\omega^{2}=g k \tanh k h$, while $k_{n}$ 's are the positive real roots of $\omega^{2}=-g k_{n} \tan k_{n} h$. The coefficients $A$ and $A_{n}$ are to be determined from Eq. (4), such that

$$
A \cosh k y+\sum_{n=1}^{\infty} A_{n} \cos k_{n} y=\xi(y)
$$

whence we have

$$
\begin{aligned}
A & =\frac{2 k \int_{0}^{n} \xi(y) \cosh k y d y}{\sinh k h \cosh k h+k h} \\
A_{n} & =\frac{2 k_{n} \int_{0}^{h} \xi(y) \cos k_{n} y d y}{\sin k_{n} h \cos k_{n} h+k_{n} h}
\end{aligned}
$$

With this complete set of coefficients defining the velocity potential, we may proceed to describe the orbital motion of an individual water particle whose mean position is given by the initial coordinates $x$ and $y$. The displacement of a water particle in the $x$ and $y$ directions are ${ }^{1)}$

$$
\begin{aligned}
X= & X_{w}+X_{i} \\
= & A \cosh k y \sin (\omega t-k x) \\
& +\sum_{n=1}^{\infty} A_{n} \cos k_{n} y \exp \left(-k_{n} x\right) \sin \omega t,
\end{aligned}
$$




$$
\begin{aligned}
Y= & Y_{w}+Y_{i} \\
= & A \sinh k y \cos (\omega t-k x) \\
& +\sum_{n=1}^{\infty} A_{n} \sin k_{n} y \exp \left(-k_{n} x\right) \sin \omega t,
\end{aligned}
$$

respectively.

It is evident that the fluid motion is composed of two parts: a wave part propagating in the positive $x$ direction, and a part due to the local disturbances, i.e., a sum of terms of which each decays exponentially as $\exp \left(-k_{n} x\right)$. Obviously, the influence of the local disturbances virtually disappears when $x>3 h$ [Ref. (1)], but their role is important to the hydrodynamic loads on the waveboard and to the wave profile distortions very near the waveboard.

In connection with Eq. (10), the wave amplitude $a$ far down the channel (say $x>3 h$ ) is ${ }^{1)}{ }^{6}$ )

$$
a=A \sinh k h \text {. }
$$

With the aid of the Bernoulli's theorem, the hydrodynamic pressure $p$ is ( $\rho$ is water density):

$$
\begin{aligned}
p= & -\rho \frac{\partial \phi}{\partial t} \\
= & \rho g \tanh k h A \cosh k y \cos (\omega t-k x) \\
& +\rho g \sum_{n=1}^{\infty} A_{n} \tan k_{n} h \cos k_{n} y \exp \left(-k_{n} x\right) \sin \omega t
\end{aligned}
$$

The formulation so far is applicable to a general form of waveboard configuration, within the framework of linearization. The problem then narrows down to the determination of $\omega, k_{n}$ 's, $A$, and $A_{n}$ 's provided that the constant water depth $h$, the desired wavelength $\lambda$, and the particulars of the waveboard motions are prescribed.

\section{Specialization to an Articulated Double-flapWavemaker}

Let us now specialize the above formulation to an articulated double-flap wavemaker of Fig. 1. The flap sizes are $f_{u}=h-h_{2}$, and $f_{L}=h_{2}-h_{1}$, for the upper and the lower flaps, respectively. The length of the overall waveboard is given by $f \equiv f_{u}$ $+f_{L}=h-h_{1}$.

From Fig. 1, the contact surface equation $\xi(y)$ is given as

$$
\xi(y)= \begin{cases}0 & \text { for } 0 \leq y<h_{1}, \\ \alpha_{1}\left(y-h_{1}\right) & \text { for } h_{1} \leq y<h_{2}, \\ \alpha_{1}\left(y-h_{1}\right)+\alpha_{2}\left(y-h_{2}\right) & \text { for } h_{2} \leq y \leq h,\end{cases}
$$

thus, either $\alpha_{1}=0$ or $\alpha_{2}=0$ corresponds to a singleflap wavemaker hinged at $y=h_{2}$ or $y=h_{1}$, respectively. In the present development, $\alpha_{1}$ is assumed to be nonzero. From Eq. (13), it follows that the waveboard stroke at the mean water level $S$ is

$$
S=\alpha_{1}\left(h-h_{1}\right)+\alpha_{2}\left(h-h_{2}\right)
$$

Substituting Eq. (13) into Eqs. (7) and (8), we have

$$
\begin{aligned}
\frac{A}{h} & =\frac{2 k h\left[\alpha_{1} U\left(H_{1}\right)+\alpha_{2} U\left(H_{2}\right)\right]}{\sinh k h \cosh k h+k h} \\
\frac{A_{n}}{h} & =\frac{2 k_{n} h\left[\alpha_{1} U_{n}\left(H_{1}\right)+\alpha_{2} U_{n}\left(H_{2}\right)\right.}{\sin k_{n} h \cos k_{n} h+k_{n} h}
\end{aligned}
$$

where $H_{1} \equiv h_{1} / h, H_{2} \equiv h_{2} / h$, and the functions $U(r)$ and $U_{n}(r)$ are defined as

$$
U(r) \equiv \frac{1-r}{k h} \sinh k h-\frac{1}{(k h)^{2}}\{\cosh k h-\cosh [(k h) r]\},
$$

$U_{n}(r) \equiv \frac{1}{\left(k_{n} h\right)^{2}}\left\{\cos k_{n} h-\cos \left[\left(k_{n} h\right) r\right]\right\}-\frac{1-r}{k_{n} h} \sin k_{n} h$.

From Eqs. (11) and (14), after some algebraic manipulation the transfer function $a / S$ (the ratio of the wave amplitude $a$ to the waveboard stroke at the mean water level $S$ ) is shown to be

$$
\begin{aligned}
a / S & =\frac{2 k h \sinh k h}{\sinh k h \cosh k h+k h} \\
& \times\left\{\frac{U^{2}\left(H_{1}\right)+\left[\frac{\alpha_{2}}{\alpha_{1}} U\left(H_{2}\right)\right]^{2}+2 \frac{\alpha_{2}}{\alpha_{1}} U\left(H_{1}\right) U\left(H_{2}\right)}{\left(1-H_{1}\right)^{2}+\left[\frac{\alpha_{2}}{\alpha_{1}}\left(1-H_{2}\right)\right]^{2}+2 \frac{\alpha_{2}}{\alpha_{1}}\left(1-H_{1}\right)\left(1-H_{2}\right)}\right\}^{1 / 2}
\end{aligned}
$$

As is in the case of as ingle-flap wavemaker, the transfer function $a / S$ takes a limiting value of 2 for very short waves $(k h \gg 1)$, regardless of the waveboard particulars.

On the other hand, the maximum waveform distortion $\eta / a$ at $x=0$, which is obtainable from the local disturbances part of Eq. (10), may be expressed as

$$
\begin{aligned}
\eta / a & =\frac{\sinh k h \cosh k h+k h}{2 k h \sinh k h} \\
& \times \sum_{n=1}^{\infty}\left[\frac{2 k_{n} h \sin k_{n} h}{\sin k_{n} h \cos k_{n} h+k_{n} h}\right. \\
& \left.\times\left\{\frac{U_{n}^{2}\left(H_{1}\right)+\left[\frac{\alpha_{2}}{\alpha_{1}} U_{n}\left(H_{2}\right)\right]^{2}+2 \frac{\alpha_{2}}{\alpha_{1}} U_{n}\left(H_{1}\right) U_{n}\left(H_{2}\right)}{U^{2}\left(H_{1}\right)+\left[\frac{\alpha_{2}}{\alpha_{1}} U\left(H_{2}\right)\right]^{2}+2 \frac{\alpha_{2}}{\alpha_{1}} U\left(H_{1}\right) U\left(H_{2}\right)}\right\}\right]
\end{aligned}
$$

Now, in order to compute the hydrodynamic load on the waveboard, the hydrodynamic pressure at $x=$ 0 is rewritten:

$$
\left.p\right|_{x=0}=p_{w} \cos \omega t+p_{i} \sin \omega t,
$$

where

$$
\begin{aligned}
p_{w} & =\rho g a\left[\frac{A}{a} \tanh k h \cosh k y\right] \\
p_{i} & =\rho g a\left[\sum_{n=1}^{\infty} \frac{A_{n}}{a} \tan k_{n} h \cos k_{n} y\right]
\end{aligned}
$$

Clearly, the wave (or normal) pressure $p_{w}$ denotes the wave part of the hydrodynamic pressure fluctuations, and $p_{i}$, designated as the inertia pressure by Biésel $^{1)}$, represents the local disturbances in the 
vicinity of the waveboard. The wave pressure $p_{w}$, in phase with the waveboard velocity, indicates that a finite amount of work is transferred over one cycle. The energy expenditure is consumed by the propagation of outgoing waves on the free surface. The inertia pressure $p_{i}$, in phase with the waveboard acceleration, does no work on the average, however, the principal role played by the inertia pressure turns up in the form of added mass effect of the waveboard motions. In the terminology of naval hydrodynamics, $p_{i}$ and $p_{w}$ are associated with the added mass and damping coefficients, respectively.

It follows that the theoretical mean power consumption of the wavemaker over one cycle, $P$, is related to $p_{w}$ only, and can thus be shown to be ${ }^{1), 7)}$

$$
P \cong B \rho g a^{2}(g / k)^{1 / 2}
$$

which is, in fact, the energy transmission to a sinusoidal wavetrain of wavelength $\lambda$, amplitude $a$, and the propagation speed $c=(g / k)^{1 / 2}$. In arriving at Eq. (22), the finite bottom effect on the wave propagation speed has been neglected for the wavelength range of practical significance in towing tank operations.

By integrating the hydrodynamic pressure at $x=0$ over the flap lengths, we calculate the pure hydrodynamic loads $F_{u}$ and $F_{L}$, acting on the upper and the lower flaps, respectively (the factor of 2 takes into account that water fills in both sides of the waveboard ${ }^{5}$ :

$$
\begin{array}{r}
\frac{F_{u}}{2(\rho g a) B h}=\left[\frac{A}{a} \frac{\tanh k h}{k h}\left(\sinh k h-\sinh k h_{2}\right)\right] \operatorname{cost} \omega t \\
+\left[\sum_{n=1}^{\infty} \frac{A_{n}}{a} \frac{\tan k_{n} h}{k_{n} h}\left(\sin k_{n} h-\sin k_{n} h_{2}\right)\right] \sin \omega t
\end{array}
$$

$$
\begin{array}{r}
\frac{F_{L}}{2(\text { gga } B h}=\left[\frac{A}{a} \frac{\tanh k h}{k h}\left(\sinh k h_{2}-\sinh k h_{1}\right)\right] \cos \omega t \\
+\left[\sum_{n=1}^{\infty} \frac{A_{n}}{a} \frac{\tan k_{n} h}{k_{n} h}\left(\sin k_{n} h_{2}-\sin k_{n} h_{1}\right)\right] \sin \omega t
\end{array}
$$

In a similar manner, the hydrodynamically-induced torques $T_{u}$ (for the upper flap $\overline{Q_{2} Q_{3}}$ ) and $T_{L}$ (for the lower flap $\overline{\left.Q_{1} Q_{2}\right)}$ about their respective hinge points $Q_{2}$ and $Q_{1}$ may be calculated as:

$$
\begin{aligned}
& \frac{T_{u}}{2 B \rho g a h^{2}}=\frac{A}{a} \tanh k h \\
& \times\left[\frac{1-H_{2}}{k h} \sinh k h-\frac{1}{(k h)^{2}}\left(\cosh k h-\cosh k h_{2}\right)\right] \cos \omega t \\
& +\sum_{n=1}^{\infty}\left\{\frac { A _ { n } } { a } \operatorname { t a n } k _ { n } h \left[\frac{1}{\left(k_{n} h\right)^{2}}\left(\cos k_{n} h-\cos k_{n} h_{2}\right)\right.\right. \\
& \left.\left.+\frac{1-H_{2}}{k_{n} h} \sin k_{n} h\right]\right\} \sin \omega t \\
& \frac{T_{L}}{2 B \rho g a h^{2}}=\frac{A}{a} \tanh k h \\
& \quad \times\left[\frac{H_{2}-H_{1}}{k h} \sinh k h_{2}\right.
\end{aligned}
$$

$$
\begin{aligned}
& \left.-\frac{1}{(k h)^{2}}\left(\cosh k h_{2}-\cosh k h_{1}\right)\right] \cos t \omega t \\
& +\sum_{n=1}^{\infty}\left\{\frac { a } { A _ { n } } \operatorname { t a n } k _ { n } h \left[\frac{1}{\left(k_{n} h\right)^{2}}\left(\cos k_{n} h_{2}-\cos k_{n} h_{1}\right)\right.\right. \\
& \left.\left.+\frac{H_{2}-H_{1}}{k_{n} h} \sin k_{n} h_{2}\right]\right\} \sin \omega t
\end{aligned}
$$

\section{Numerical Computation and Discussion}

In the wavelength range of practical usefulness to towing tank operation, $0.1<\lambda / h<4.0$, numerical computations are carried out to investigate the dynamic characteristics of articulated double-flap wavemakers. In the initial design stages of a double-flap wavemaker, the entire waveboard length and the waveboard partition may be considered as variable parameters. Once a double-flap wavemaker is installed, this implies that $f / h, f_{u} / h$, and $f_{L} / h$ are fixed, and only $\omega, \alpha_{1}$, and $\alpha_{2} / \alpha_{1}$ are the parameters that can be varied during operations. Basically, in contrast to a single-flap wavemaker $\left(\alpha_{2} / \alpha_{1}=0\right)$, a double-flap wavemaker has an additional degree of freedom, i.e., $\alpha_{2} / \alpha_{1} \neq 0$, which is essentially the primary source of the added versatility of a doubleflap wavemaker.

Fig. 2 shows a typical plot of the transfer function $a / S$ of an articulated double-flap wavemaker (flap sizes are $f_{u} / h=0.2, f_{L} / h=0.3$ in this example). Note that the curve of $\alpha_{2} / \alpha_{1}=0.0$ corresponds to the transfer function of a single-flap wavemaker ${ }^{2), 3)}$ of flap length $f_{S} / h=f / h=0.5$. The variation of $a / S$ with respect to the waveboard partition, i.e., the location of the upper hinge point $\mathrm{H}_{2}$, is displayed in Fig. 3, when the overall waveboard length is $f / h=0.5$ and the flap angle ratio $\alpha_{2} / \alpha_{1}=5.0$. Figs. 2 and 3 give support to the intuitively obvious predictions of the $\alpha / S$-variation, that is, higher values of $\alpha / S$ would be attained if a greater amount of

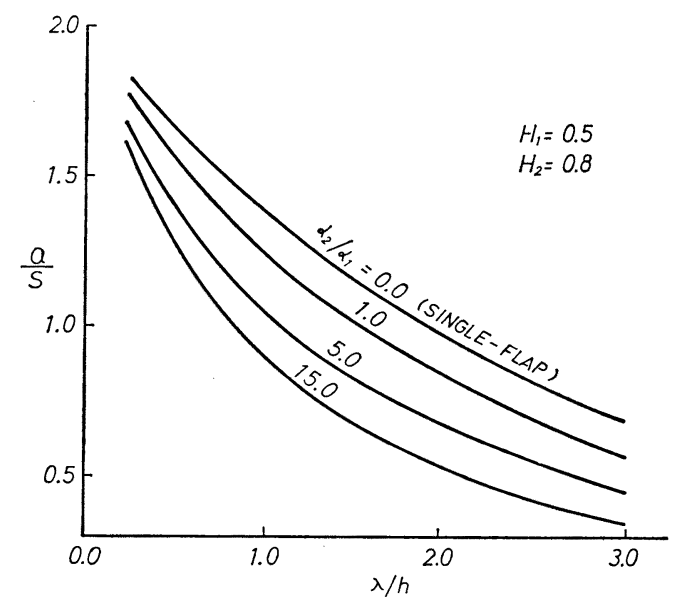

Fig. 2 Variation of the transfer function $a / S$ as functions of $\lambda / h$ and $\alpha_{2} / \alpha_{1}$. Flap sizes are $f_{u} / h=0.2, f_{L} / h=0.3$. 


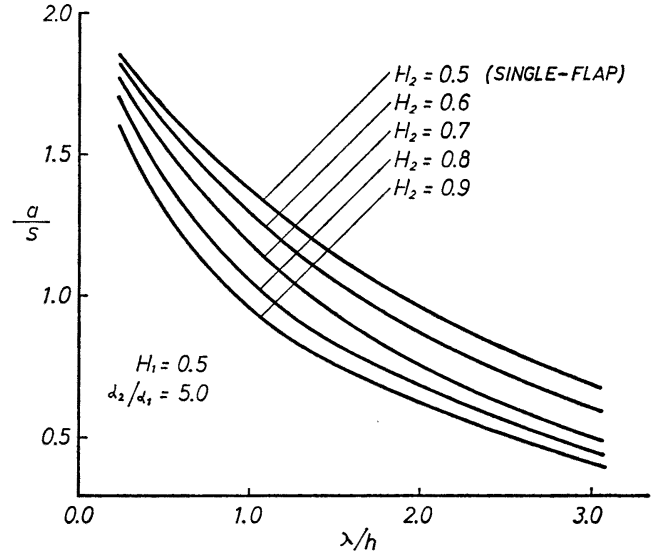

Fig. 3 Variation of the transfer function $\alpha / S$ as functions of $\lambda / h$ and $H_{2}$. Overall waveboard length is $f / h=0.5$, and the flap angle ratio is $\alpha_{2} / \alpha_{1}=5.0$.

water is accelerated by the waveboard movement. This, in turn, suggests that for a given waveboard length $f / h$, the upper flap size $f_{u} / h$ should be larger and the flap angle ratio $\alpha_{2} / \alpha_{1}$ should be smaller, in order to obtain higher values of $a / S$. In other words, as long as the transfer function $a / S$ alone is concerned, a single-flap wavemaker $\left(\alpha_{2} / \alpha_{1}=0\right)$ is most effective for a given waveboard length.

Fig. 4 illustrates the variation of the wave pressure $p_{w}$ with respect to the wavelength. At the same time, if the abscissa of Fig. 4 is replaced by $X_{w} / a$ of Eq. (9), then it will also serve as the envelope of the maximum particle excursions in the $x$ direction far down the channel. The wave pressure $p_{w}$ is clearly the hydrodynamic pressure far down the channel, which is independent of the waveboard particulars. As is well-known, in the case of short waves $p_{w}$ (or $X_{w}$ ) is appreciable only in a narrow layer adjacent to the surface and decays rapidly with increasing water depth. As the wavelength increases, $p_{w}$ (or $X_{w}$ ) is felt into in-

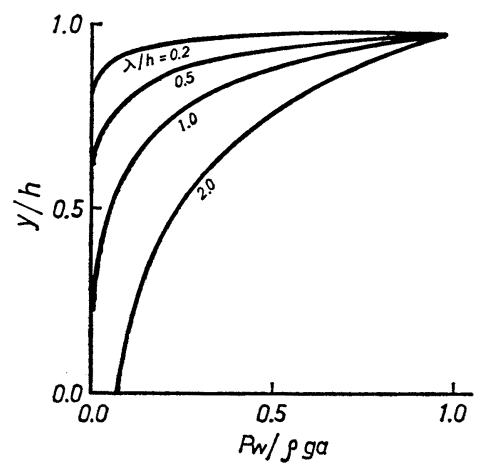

Fig. 4 Plot of the wave pressure $p_{w}$ vs. water depth. creasing water depths, making the finite bottom effect nonnegligible.

Let us now turn to the question of the local disturbances, a sum of terms of which each decays exponentially as $\exp \left(-k_{n} x\right)$. For a given $k h$, there exist an infinite number of $k_{n} h$ 's, but the first few terms are of dominant importance $\left.{ }^{1)}, 6\right)$. In the present numerical computations, the series was terminated up to the first fifty terms to insure sufficient accuracy ${ }^{5}$.

In contrast to the wave pressure $p_{w}$, the inertia pressure $p_{i}$ displays marked dependence on the waveboard particulars as well as on wavelength. In Figs. 5 7, plots of the inertia pressure distribution are shown for varying design parameters. The following two points can readily be noticed: (1) unlike $p_{w}$ which decays with increasing water depth, $p_{i}$ sustains its presence throughout much of the water layer on the whole, (2) in the case of moderate and long waves, i.e., $\lambda / h \geq 2.0$, the overall magnitude of $p_{i}$ is relatively small, however, $p_{i}$ is immensely augmented when short waves are to be generated. From Figs. $5 \sim 7$, it is found that, in the case of short waves (e.g., $\lambda / h=0.25$ ), employing a double-flap waveboard with large articulation (e.g., $\alpha_{2} / \alpha_{1}=5.0$ ) has a noticeable effect in cutting

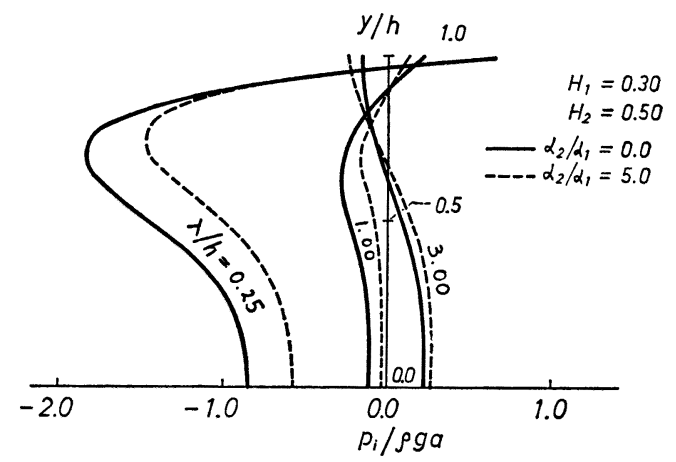

Fig. 5 Plot of the inertia pressure $p_{i}$ vs. water depth. Flap sizes are $f_{u} / h=0.5, f_{L}=0.2$, respectively.

down the inertia pressure. It is also interesting to note in Fig. 5 that, in the case of long waves (e.g., $\lambda / h=3.00), p_{i}$ is slightly enlarged by use of a double-flap waveboard (e.g., $\alpha_{2} / \alpha_{1}=5.0$ ) This is an example of improperly utilized double-flap wavemakers.

As was pointed out earlier, the inertia pressure $p_{i}$ arises because of the local disturbances in the immediate vicinity of the waveboard, which are created mostly by the differences between the waveboard motions and the actual water particle movements in waves. Accordingly, within the framework of linearized formulation, one crude way of making a rough estimate of the magnitude of the inertia pressure is to examine the difference between 
$\xi(y) / a$ of Eq. (13) and the envelope of the maximum particle excursions in the $x$ direction, that is, Fig. 4 with its abscissa replaced by $X_{w} / a$. Consequently, with an eye to reducing the inertia pressure, the following observations may be made: In the case of short waves (e.g., $\lambda / h=0.25$ ) the curve of $X_{w} / a$ decays rapidly with increasing water depth, which suggests that the overall waveboard length $f / h$ should be small and the flap angle ratio $\alpha_{2} / \alpha_{1}$ should be large. However, it should be remembered that all the arguments here are within the scope of linearized small-amplitude motions; therefore, excessively large values of $\alpha_{2} / \alpha_{1}$, which would violate the underlying assumption that the flap angles are small, are excluded from the analysis. In the opposite situation, when moderate and long waves (e.g., $\lambda / h \geq 2.0$ ) are generated, the $X_{w} / a$ curve decays much more mildly, thus a single-flap waveboard $\left(\alpha_{2} / \alpha_{1}=0\right)$ of increased sizes is more appropriate. In the limit of extremely long waves $(\lambda / h \gg 1)$, a piston-type wavemaker $(f / h=1.00)$ with uniform horizontal stroke will be preferable.

The practical advantages of an articulated doubleflap wavemaker is manifested by evaluating the wavemaker performance, such as the waveform distortion very near the waveboard and the hydrodynamic loads exerted on the waveboard, especially in the range of short waves (say $\lambda / h \leqq 1.0$ ).

The maximum waveform distortion $\eta / a$ at $x=0$, derived in Eq. (20), is shown in Table 1, for the case when the overall waveboard length is $f / h=$ 0.5 . As to the short waves $(\lambda / h \leqq 1.0)$, the waveform distortion $\eta / a$ diminishes as the upper flap size becomes smaller and the flap angle ratio increases. This is an expected result in view of the effectiveness of a double-flap wavemaker in reducing the inertia pressure (or the local disturbances), as was illustrated in Figs. 5 7. However, in the case

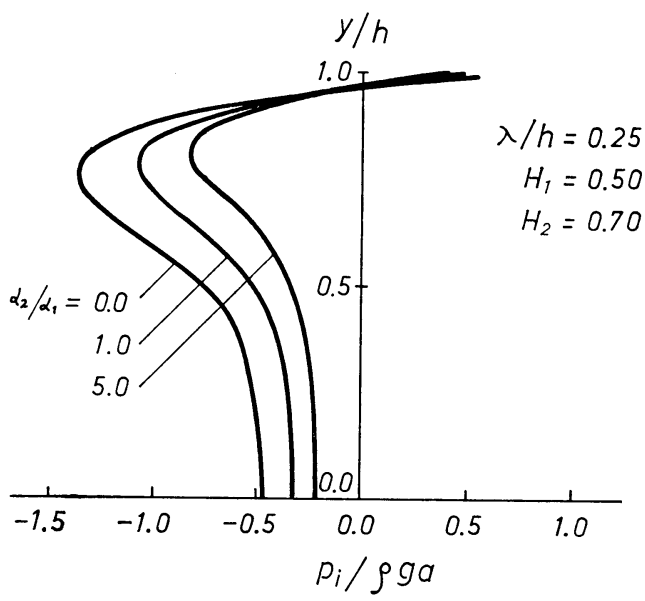

Fig. 6 Plot of the inertia pressure $p_{i}$ vs. water depth, for the case of $\lambda / h=0.25$. Flap sizes are $f_{u} / h=0.3, f_{L} / h=0.2$.

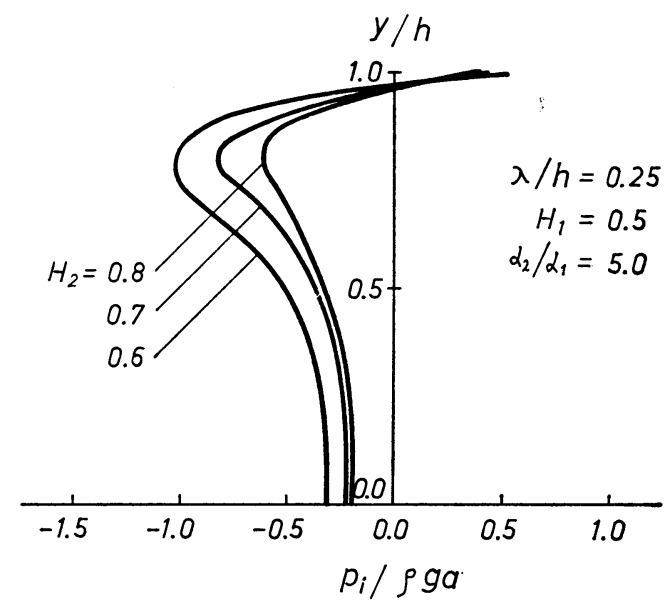

Fig. 7 Plot of the inertia pressure $p_{i}$ vs. water depth, for the case of $\lambda / h=0.25, f / h=$ $0.5, \alpha_{2} / \alpha_{1}=5.0$.

of moderate and long waves (e.g., $\lambda / h=2.0$ ), the waveform distortion $\eta / a$ actually increases by use of double-flap wavemakers. These findings are in line with our previous discussion that a single-flap wavemaker $\left(\alpha_{2} / \alpha_{1}=0\right)$ with large waveboard lengths $(f / h \rightarrow 1.0)$ is suitable for the generation of mederate and long waves. Obviously, if a single-flap wavemaker equipped with a waveboard of much larger size were utilized, then $\eta / a$ would be much smaller for moderate and long waves. In summary, Table 1 indicates that for a given waveboard geometry, in order to attain small values of $\eta / a$, the flap angle ratio $\alpha_{2} / \alpha_{1}$ should be chosen roughly along the diagonal line, stretching from lower left to upper right corner, of Table 1 .

The total hydrodynamic force acting on the entire waveboard $F\left(=F_{u}+F_{L}\right)$, of Eqs. (23) and (24), is shown in Table 2 , for the case when $f / h=0.5$. Here $F$, nondimensionalized by $2 B h \rho g a$, is expressed in the form of $M \cos (\omega t-\beta)$. It is seen in Table 2 that the capability of double-flap wavemakers in reducing the hydrodynamic load $F$ is pronounced for short waves. For instance, in the example of $\lambda / h=0.25$, use of a double-flap wavemaker (e.g., $H_{2}=0.8, \alpha_{2} / \alpha_{1}=5.0$ ) cuts down the hydrodynamic load $F$ more than half in comparison with the single-flap wavemaker $\left(\alpha_{2} / \alpha_{1}=0.0\right)$ with the same waveboard size $f / h=0.5$. However, in the case of moderate and long waves (e.g., $\lambda / h \geq 2.0$ ) not much reduction is achieved in hydrodynamic load $F$ by use of double-flap wavemakers.

As was seen before, when short waves are generated, $p_{i}$ overwhelms $p_{w}$ (the phase angle $\beta$ of Table 2 is close to $-\pi / 2$ ), therefore, a significant reduction in terms of hydrodynamic loads is anticipated by use of double-flap wavemakers. On the contrary, in the case of moderate and long waves, $p_{w}$ is the 
Table 1. Maximum waveform distortion $r / a$ at $x=0$, as computed from Eq. (21).

\begin{tabular}{l|c|c|c|c|c}
\hline \hline & & $\alpha_{2} / \alpha_{1}=0.0$ & $\alpha_{2} / \alpha_{1}=0.5$ & $\alpha_{2} / \alpha_{1}=1.0$ & $\alpha_{2} / \alpha_{1}=5.0$ \\
\hline & $\lambda / h=0.25$ & 0.5751 & 0.5533 & 0.5411 & 0.5135 \\
$H_{1}=0.5$ & $\lambda / h=0.50$ & 0.3839 & 0.3612 & 0.3483 & 0.3188 \\
$H_{2}=0.6$ & $\lambda / h=1.00$ & 0.1428 & 0.1205 & 0.1076 & 0.0773 \\
& $\lambda / h=2.00$ & 0.1317 & 0.1531 & 0.1658 & 0.1960 \\
\hline & $\lambda / h=0.25$ & 0.5751 & 0.5348 & 0.5090 & 0.4401 \\
$H_{1}=0.5$ & $\lambda / h=0.50$ & 0.3839 & 0.3441 & 0.3181 & 0.2458 \\
$H_{2}=0.7$ & $\lambda / h=1.00$ & 0.1428 & 0.1064 & 0.0818 & 0.0095 \\
& $\lambda / h=2.00$ & 0.1317 & 0.1645 & 0.1875 & 0.2587 \\
\hline & $\lambda / h=0.25$ & 0.5751 & 0.5267 & 0.4908 & 0.3679 \\
$H_{1}=0.5$ & $\lambda / h=0.50$ & 0.3839 & 0.3396 & 0.3058 & 0.1808 \\
$H_{2}=0.8$ & $\lambda / h=1.00$ & 0.1428 & 0.1062 & 0.0769 & 0.0409 \\
& $\lambda / h=2.00$ & 0.1317 & 0.1623 & 0.1877 & 0.2975
\end{tabular}

Table 2. Total hydrodynamic load $F\left(F_{u}+F_{L}\right)$ acting on the entire waveboard $\left(H_{1} \leq y / \leq 1.0\right)$, nondimensionalized by $2 B \rho g a h$. Quantities are expressed in the form of $M(\cos (\omega t-\beta)$.

\begin{tabular}{|c|c|c|c|c|c|c|c|c|}
\hline & \multicolumn{2}{|c|}{$\alpha_{2} / \alpha_{1}=0.0$} & \multicolumn{2}{|c|}{$\alpha_{2} / \alpha_{1}=0.5$} & \multicolumn{2}{|c|}{$\alpha_{2} / \alpha_{1}=1.0$} & \multicolumn{2}{|c|}{$\alpha_{2} / \alpha_{1}=5.0$} \\
\hline & $M$ & $\beta$ & $M$ & $\beta$ & $M$ & $\beta$ & $M$ & $\beta$ \\
\hline & & & \multicolumn{2}{|c|}{$H_{1}=0.5, H_{2}=0.6$} & & & & \\
\hline$\lambda / h=0.25$ & 0.4830 & -1.4883 & 0.4407 & -1.4804 & 0.4169 & -1.4752 & 0.3663 & -1.4810 \\
\hline$\lambda / h=0.50$ & 0.1873 & -1.1329 & 0.1708 & -1.0870 & 0.1616 & -1.0568 & 0.1411 & -0.9729 \\
\hline$\lambda / h=1.00$ & 0.1545 & -0.1702 & 0.1535 & -0.1258 & 0.1531 & -0.0998 & 0.1524 & -0.0385 \\
\hline$\lambda / h=2.00$ & 0.2540 & -0.0170 & 0.2539 & -0.0094 & 0.2539 & -0.0049 & 0.2539 & -0.0060 \\
\hline \multirow[t]{2}{*}{$\lambda / h=3.00$} & 0.3205 & -0.1138 & 0.3205 & -0.1122 & 0.3205 & -0.1113 & 0.3204 & -0.1091 \\
\hline & & & \multicolumn{2}{|c|}{$H_{1}=0.5, H_{2}=0.7$} & & & & \\
\hline$\lambda / h=0.25$ & 0.4830 & -1.4883 & 0.4150 & -1.4757 & 0.3781 & -1.4654 & 0.2689 & -1.4223 \\
\hline$\lambda / h=0.50$ & 0.1873 & -1.1329 & 0.1634 & -1.0632 & 0.1483 & -1.0056 & 0.1103 & -0.7666 \\
\hline$\lambda / h=1.00$ & 0.1545 & -0.1702 & 0.1532 & -0.1083 & 0.1526 & -0.0659 & 0.1526 & 0.0595 \\
\hline$\lambda / h=2.00$ & 0.2540 & -0.0170 & 0.2539 & -0.0063 & 0.2539 & 0.0013 & 0.2540 & 0.0247 \\
\hline \multirow[t]{2}{*}{$\lambda / h=3.00$} & 0.3205 & -0.1138 & 0.3205 & -0.1111 & 0.3204 & -0.1092 & 0.3205 & -0.1031 \\
\hline & & & \multicolumn{2}{|c|}{$H_{1}=0.5, H_{2}=0.8$} & & & & \\
\hline$\lambda / h=0.25$ & 0.4830 & -1.4883 & 0.4224 & -1.4765 & 0.3778 & -1.4652 & 0.2248 & -1.3929 \\
\hline$\lambda / h=0.50$ & 0.1873 & -1.1329 & 0.1664 & -1.0731 & 0.1508 & -1.0160 & 0.1108 & -0.6629 \\
\hline$\lambda / h=1.00$ & 0.1545 & -0.1702 & 0.1534 & -0.1203 & 0.1528 & -0.0798 & 0.1523 & 0.0840 \\
\hline$\lambda / h=2.00$ & 0.2540 & -0.0170 & 0.2539 & -0.0085 & 0.2539 & -0.0014 & 0.2540 & 0.0247 \\
\hline$\lambda / h=3.00$ & 0.3205 & -0.1138 & 0.3205 & -0.1114 & 0.3204 & -0.1095 & 0.3201 & -0.1006 \\
\hline
\end{tabular}

dominant contributor to the hydrodynamic loads (the phase angle $\beta$ of Table 2 is close to zero). In this situation, use of double-flap wavemakers, which can affect only $p_{i}$ does not lead to an appreciable reduction in the hydrodynamic loads.

Finally, Table 3 contains numerical examples of the hydrodynamically-induced torques $T_{u}$ (for the upper flap $\overline{Q_{2} Q_{3}}$ ) and $T_{L}$ (for the lower flap $\overline{Q_{1} Q_{2}}$ ), of Eqs. (25) and (26), when the flap sizes are $f_{u} / h=0.2$ and $f_{L} / h=0.3$. In Table 3 , the torques are made dimensionless by $2 B h^{2} \rho g a$, and is expressed in the form of $M \cos (\omega t-\beta)$. First, let us consider short waves (e.g., $\lambda / h=0.25$ ). We can see that the wave pressure $p_{w}$ is still largely unattenuated in the region of the upper flap $\overline{Q_{2} Q_{3}}(0.8 \leq y / h \leq 1.0)$. Therefore, is composed of contributions from both the wave pressure and the inertia pressure (the phase angle $\beta$ assumes values roughly half-way between zero and $-\pi / 2)$. On the other hand, since the wave pressure $p_{w}$ is rapidly decaying with in- 
Table 3. Hydrodynamically-induced torques $T_{u}$ (for the upper flap $\overline{Q_{2} Q_{3}}$ and $T_{L}$ (for the lower flap $\overline{Q_{1} Q_{2}}$ ), nondimensionalized by $2 B \rho g a h^{2}$. Quantities are expressed in the form of $M \cos (\omega t-\beta)$. Flap sizes are $f_{u} / h=0.2, f_{L} / h=0.3$.

\begin{tabular}{|c|c|c|c|c|c|c|c|c|c|}
\hline & & \multicolumn{2}{|c|}{$\alpha_{2} / \alpha_{1}=0.0$} & \multicolumn{2}{|c|}{$\alpha_{2} / \alpha_{1}=0.5$} & \multicolumn{2}{|c|}{$\alpha_{2} / \alpha_{1}=1.0$} & \multicolumn{2}{|c|}{$\alpha_{2} / \alpha_{1}=5.0$} \\
\hline & & $M$ & $\beta$ & $M$ & $\beta$ & $M$ & $\beta$ & $M$ & $\beta$ \\
\hline \multirow{2}{*}{$\lambda / h=0.25$} & $T_{U}$ & 0.0096 & -0.8449 & 0.0091 & -0.7967 & 0.0088 & -0.7576 & 0.0077 & -0.5989 \\
\hline & $T_{L}$ & 0.0564 & 1.5708 & 0.0488 & 1.5708 & 0.0432 & 1.5708 & 0.0239 & 1.5708 \\
\hline \multirow{2}{*}{$\lambda / h=0.50$} & $T_{U}$ & 0.0101 & 0.0291 & 0.0101 & 0.0225 & 0.0101 & 0.0173 & 0.0101 & -0.0015 \\
\hline & $T_{L}$ & 0.0230 & 1.5081 & 0.0197 & 1.4981 & 0.0172 & 1.4881 & 0.0080 & 1.3923 \\
\hline \multirow{2}{*}{$\lambda / h=1.00$} & $T_{U}$ & 0.0138 & 0.0772 & 0.0137 & 0.0547 & 0.0137 & 0.0366 & 0.0137 & -0.0363 \\
\hline & $T_{L}$ & 0.0089 & -0.5801 & 0.0083 & -0.4383 & 0.0078 & -0.3085 & 0.0078 & 0.2752 \\
\hline \multirow{2}{*}{$\lambda / h=2.00$} & $T_{U}$ & 0.0166 & -0.1308 & 0.0166 & -0.1522 & 0.0167 & -0.1699 & 0.0169 & -0.2451 \\
\hline & $T_{L}$ & 0.0182 & 0.0343 & 0.0182 & 0.0709 & 0.0182 & 0.1018 & 0.0186 & 0.2332 \\
\hline \multirow{2}{*}{$\lambda / h=3.00$} & $T_{U}$ & 0.0183 & -0.2805 & 0.0184 & -0.3004 & 0.0185 & -0.3169 & 0.0190 & -0.3888 \\
\hline & $T_{\boldsymbol{L}}$ & 0.0252 & -0.0450 & 0.0252 & -0.0244 & 0.0252 & -0.0077 & 0.0252 & 0.0693 \\
\hline
\end{tabular}

creasing water depth for short waves, $p_{w}$ is very small in the region of the lower flap $\overline{Q_{1} Q_{2}}(0.5 \leq y)$ $h \leq 0.8$ ). Hence $T_{L}$ is determined principally by the inertia pressure $p_{i}$ (the phase angle $\beta$ is close to $\pi / 2)$. For this reason, double-flap wavemakers are seen to be more effective in reducing $T_{L}$, rather than $T_{u}$, in the case of short waves. Secondly, we turn to discuss the cases of moderate and long waves. The dominant contributor to the hydrodynamically-induced torques is the wave pressure $p_{w}$, which is not affected by the changes in waveboard particulars. Here again we see that a singleflap wavemaker with large waveboard lengths is desirable from the standpoint of having small torques.

Table 3 suggests that the setting of the operational variable $\alpha_{2} / \alpha_{1}$ should preferably be selected from roughly along the diagonal line of Table 3 , from lower left to upper right corner. This is a similar result that we arrived at in Table 1 , with a view towards having smallest waveform distortions.

\section{Conclusion}

A theoretical account is given of an articulated double-flap wavemaker, as a sequel to the previous paper $^{2)}$ concerning the usual single-flap wavemaker. The additional degree of freedom in double-flap wavemakers is capable of reducing the local disturbances in the vicinity of the waveboard, which turns up in the form of much decreased inertia pressure especially in the case of short waves. Therefore, when short waves are to be generated, it is desirable that the overall waveboard length $f / h$ be small and the flap angle ratio $\alpha_{2} / \alpha_{1}$ be relatively large. On the other hand, in the case of moderate and long waves, larger waveboards $(f / h \rightarrow 1.0)$ with little or zero flap angle ratio $\left(\alpha_{2} / \alpha_{1} \rightarrow 0\right)$ are preferable.
The analysis clearly suggests that considerable improvement over single-flap wavemakers in performance curves, such as waveform distortion near the waveboard and the hydrodynamic loads, is feasible by suitable use of double-flap waveboards.

Needless to say, the results of the present study should be the subject of careful experimental verification.

\section{References}

1) Biésel, F., "Laboratory Wave-Generating Apparatus," English Translation in Project Report 39, St. Anthony Falls Hydraulic Lab., Univ. of Minnesota, Minneapolis, Minn., March 1953.

2) Hyun, J. M., “Theory for Hinged Wavemakers of Finite Draft in Water of Constant Depth," Journal of Hydronautics, Vol. 10, Jan. 1976, pp. 2-7.

3) Gilbert, G., Thompson, D. M., and Brewer, A.J., " Design Curves for Regular and Random Wave Generator," Journal of Hydraulic Research, Vol. 9, No. 2, 1971, pp. 163-196.

4) Hyun, J.M., "Simplified Analysis for a Plunger-type Wavemaker Performance," Journal of Hydronautics, Vol. 10, July 1976 (in press).

5) Taniguchi, K. and Kasai, H., "A New Flaptype Wavemaker without Water on Back Side," Proc. Fall Meeting of the Society of Naval Architects of Japan, 1972, pp. 129-136.

6) Ursell, F., Dean, R. G., and Yu, Y.S., "Forced Small-amplitude Water Waves: A Comparison of Theory and Experiment," Journal of Fluid Mechanics, Vol. 7, Part 1, 1959, pp. 33-52.

7) Taniguchi, K. and Shibata, J., "Wavemaker of Mitsubishi Nagasaki Experimental Tank," Publication of the Yugoslav Ship Hydro. Inst., No. 9, Zagreb, Yugoslavia, Sept. 1960, pp. (17-1)-(17-23). 